\title{
The Patient Perspective on Living with Acute Myeloid Leukemia
}

Erin L. Tomaszewski · Catherine E. Fickley $\cdot$ LeAnne Maddux $\cdot$ Robert Krupnick ·

Erkut Bahceci · Jean Paty · Floortje van Nooten

Received: April 12, 2016 / Published online: September 2, 2016

(C) The Author(s) 2016. This article is published with open access at Springerlink.com

\section{ABSTRACT}

Introduction: Acute myeloid leukemia (AML) imposes significant burden on patients, their families, and the healthcare system. Published literature has reported many AML signs and symptoms, as well as their impact on patients.

Enhanced content To view enhanced content for this article go to http://www.medengine.com/Redeem/ 0295F0606245BD20.

Electronic supplementary material The online version of this article (doi:10.1007/s40487-016-0029-8) contains supplementary material, which is available to authorized users.

E. L. Tomaszewski

University of Pittsburgh, Pittsburgh, PA, USA

C. E. Fickley · J. Paty

Quintiles, New York, NY, USA

L. Maddux · R. Krupnick

Quintiles, Cambridge, MA, USA

E. Bahceci

Astellas Pharma Global Development Inc,

Northbrook, IL, USA

F. van Nooten

Astellas Pharma Europe B.V., Leiden,

The Netherlands

Present Address:

F. van Nooten $(\varangle)$

Dompé Farmaceutici S.p.A., Milan, Italy

e-mail: floortjevannooten@hotmail.com
However, there are no publications on the experience of living with AML from the patient's perspective. In this study, we performed qualitative interviews with patients with AML to understand their experience.

Methods: Participants were recruited from the US and Japan. All patients were screened to assess eligibility, and were divided into four subgroups (i.e., newly-diagnosed, high-intensity chemotherapy; newly-diagnosed, low-intensity chemotherapy; relapse/refractory; and post-transplant). Patients were interviewed over the phone by a trained researcher and asked about their day-to-day experience with AML. Signs/ symptoms and impacts were coded, analyzed using Atlas.ti software, and reported as frequencies, with the medians of patient-reported disturbance levels (0-10) computed for each symptom and impact.

Results: The most commonly reported sign/ symptom in the US was fatigue (95.7\%), followed by bruising and weakness (both $78.3 \%)$, and in Japan, nausea (94.4\%), followed by fatigue and headache (both 88.9\%). The most commonly reported impact in the US was a decreased ability to maintain 
social/familial roles (91.3\%), followed by anxiety and a decreased ability to function (both $87.0 \%$ ), and most commonly reported in Japan was anxiety, a decreased ability to function, and remission uncertainty (94.4\%).

Conclusion: Although the frequency of signs/ symptoms and their level of disturbance varied between the US and Japan, there was remarkable consistency in the types of signs/ symptoms and impacts reported across all patients. The consistency in the experience of the disease across patients suggests that measurement of AML experience can be achieved by using the same tool for most, if not all, of these patients.

Funding: Astellas Pharma Inc., Northbrook, IL, USA.

Keywords: Acute myeloid leukemia; Patient experience; Patient-reported outcomes

\section{INTRODUCTION}

Acute myeloid leukemia (AML) is characterized by an overproduction of immature white blood cells in the bone marrow, which eventually spreads to the blood and sometimes other organs [1]. AML is a rare disease with incidence rates of 3-5 cases per 100,000 people in the United States (US) and Europe [2] and a life expectancy of 3-34 months [3] in newly-diagnosed patients. It is associated with significant treatment costs, placing a burden on the healthcare system [4]. However, the overall burden of illness on these patients and their families may be challenging to quantify.

In addition to being a rapidly progressing disease, AML appears to be quite symptomatic $[2,5-8]$, with the key signs/symptoms reported as dizziness, fatigue, fever, and weakness in literature [8-11]. Furthermore, these signs/symptoms, along with the diagnosis of a life-threatening disease, have an impact on the patients' lives, including increased anxiety, fear, hopelessness, decreased ability to maintain social/familial roles, depression, low sex drive, and remission uncertainty, among others [7, 12-14]. The signs, symptoms, and impacts, collectively called 'concepts,' are also reflected in leukemia-specific patient-reported outcome (PROs) measure instruments, which contain a number of items for patients to complete [e.g., the Functional Assessment of Cancer Therapy-Leukemia (FACT-Leu)]. Currently, it is unknown whether the experience of AML varies considerably in patients across disease/treatment stages or whether there are cultural variations of concepts. Thus, one PRO instrument may not fit all patients with AML, thereby making the measurement of the patient experience quite challenging.

To better understand this variability, we aimed at capturing the patient's experience of living with AML directly through qualitative interviews across two geographic locations, the US and Japan, and four different subgroups of patients with AML (newly-diagnosed, high-intensity chemotherapy; newly-diagnosed, low-intensity chemotherapy; relapsed/refractory; post-transplant). These countries and groups were selected to align with the regions and treatment groups included in the ongoing Astellas clinical program. At the study initiation in September 2014, a literature review revealed there are no published studies on the experience of living with AML based on patients' interviews. Thus, this study uniquely adds to our understanding of the experience of living with AML assessed from the patient's perspective. Specifically, we are now able to describe the disease using patient language, rather than being limited to our clinical/medical terminology, which reflects a unique point of view regarding AML. 


\section{METHODS}

\section{Participant Recruitment}

Potential study participants in the US were recruited in two different ways: via advertisements on the internet at AML-specific online websites and forums, or from an existing online AML community. Advertisements were interactive; by clicking on a link, potential participants could learn more about the study and provide their email address to be contacted for screening and informed consent. Once an e-mail address was provided, an e-mail with a link was sent to the potential participant. If a potential participant opted to click on the link, he or she was directed to a site and asked to complete an online screening survey and provide electronic informed consent. Potential participants who were eligible for the study and provided informed consent were contacted for scheduling of an interview. Recruitment of patients with AML who were part of an existing online community and who had previously consented to be contacted for research purposes were contacted via e-mail; these patients were then screened and consented using the same methods as described above. The study was reviewed and approved by the Schulman Institutional Review Board in the US.

Participants in Japan were recruited via clinicians who treat patients with AML. Participants were screened for additional inclusion/exclusion criteria and for willingness and ability to participate. Consent was obtained from each participant prior to the interview.

All procedures performed in the studies involving human participants were in accordance with the ethical standards of the institutional and/or national research committee and with the 1964 Helsinki declaration and its later amendments or comparable ethical standards.

\section{Participant Sample}

Participants were eligible for inclusion in the study if they lived in the US (English-speaking) or Japan (Japanese-speaking), were 18 years of age or older, and had a confirmed AML diagnosis (patients may also have had myelodysplastic syndrome) and no other blood cancer or leukemia. Patients were excluded if they had the following comorbidities: congestive heart failure, uncontrolled infection, human immunodeficiency virus, active hepatitis $\mathrm{B} / \mathrm{C}$ or other active hepatic disorder, or if they had received major surgery or radiation in the past 4 weeks. The US participants self-reported AML via the screening tool that was completed online, and thus, were not clinician-confirmed. For participants in Japan, their AML diagnosis was clinician-confirmed.

The subgroup criteria are summarized in Table 1. Participants in both countries met the criteria for one of the following four subgroups:

- Newly-diagnosed participants receiving high-intensity chemotherapy treatment (chemotherapy that includes approximately 1-month hospitalization).

- Newly-diagnosed participants receiving low-intensity chemotherapy treatment (chemotherapy that involves short-term hospitalization with a less intensive, less toxic, or lower-dose approach). These patients were $65+$ years of age, as older patients are most likely unable to tolerate high-intensity chemotherapy.

- Relapsed/refractory participants. Relapsed participants were classified as achieving remission with their first chemotherapy, but symptoms have returned. Thus, these 
participants must undergo a second round of chemotherapy. Refractory participants were those who completed their second round of chemotherapy and in whom both the first and second rounds were unsuccessful in achieving remission. Relapsed/refractory participants could have been receiving high- or low-intensity treatment.

- Post-bone marrow or stem cell transplant participants were included in the transplant subgroup regardless of whether they were newly-diagnosed or considered relapsed/ refractory.

The sample size for the study was based on achieving 'saturation,' defined as the point at which no new relevant information emerges that would contribute to understanding the patient experience when assessed across all participants [15]. To determine saturation, the sample in each country was chronologically divided into three waves of relatively equal numbers of participants, such that waves could be compared for sign/symptom and impact similarity. A similar procedure was used to evaluate saturation within participant subgroups.

\section{Participant Interview Procedures}

Individual patient interviews were performed by telephone ranging from 60 to 75 minutes in duration. The interviews were recorded and then transcribed. The trained moderators who conducted the interviews followed an interview guide based on the information extracted from published literature on the signs, symptoms, and impacts of AML and its treatments and confirmed by clinicians [15]. In Japan, a third-party vendor translated all materials before conducting the interviews. The interviewers in Japan received 2 hours' training with the study's lead moderator to discuss the key objectives of the interview. Symptoms were defined to patients as "any symptoms or sensations that you have experienced since being diagnosed with AML,"

Table 1 Categorization of four patient subgroups interviewed

\begin{tabular}{ll}
\hline Criteria & Subgroup \\
\hline $\begin{array}{l}\text { Currently receiving high-intensity chemotherapy for the first time since } \\
\text { diagnosis }\end{array}$ & $\begin{array}{c}\text { Qualified as newly-diagnosed, high-intensity } \\
\text { chemotherapy age }>18 \text { years old }\end{array}$ \\
$\begin{array}{l}\text { Recently completed your first high-intensity chemotherapy since diagnosis, } \\
\text { and the symptoms have not returned; this is called remission }\end{array}$ & $\begin{array}{l}\text { Currently receiving low-intensity chemotherapy for the first time since } \\
\text { diagnosis }\end{array}$ \\
$\begin{array}{l}\text { Recently completed your first low-intensity chemotherapy since diagnosis, } \\
\text { and the symptoms have not returned; this is called remission }\end{array}$ & $\begin{array}{c}\text { chemotherapy age } \geq 65 \text { years old } \\
\text { You achieved remission with your first chemotherapy but your symptoms } \\
\text { have returned. So you are preparing for, or receiving, or recently } \\
\text { completed your second chemotherapy }\end{array}$ \\
$\begin{array}{l}\text { You recently completed your second chemotherapy and both your first } \\
\text { and second therapies were unsuccessful in achieving remission }\end{array}$ & $\begin{array}{l}\text { Qualified as refractory age }>18 \text { years old } \\
\text { Recently completed transplant therapy }\end{array}$ \\
\hline
\end{tabular}


and impacts were described as "the kinds of impacts or effects that having the symptoms and/or side-effects mentioned have on your life. These might include effects on your daily activities, work, leisure, or social life, as well as financial and emotional effects." Thus, the signs/symptoms and impacts were those experienced by patients living with AML.

The interview itself had two components: first was an open-ended portion where the subject was encouraged to spontaneously report on their experience with AML; second was a closed-ended series of questions (probing questions) to capture details on signs, symptoms, and the impact of these on the patients' lives. Subjects were also asked to rate the disturbance level of the signs, symptoms, and impacts using an 11-point numeric rating scale ranging from 0 to 10 with 0 meaning "does not disturb" and 10 meaning "greatly disturbing."

\section{Analysis}

The qualitative interview data (i.e., interview transcripts) were analyzed using content analysis and Atlas.Ti software. We used the patients' verbal reports to create quantitative summaries of the types of signs/symptoms and the impacts that patients reported. A custom code book was created for this analysis based on the participants' demographics, information about their AML diagnosis and treatment, personal descriptions of AML, and the sign/ symptom and impact data capture forms utilized in the qualitative interviews. These forms contained lists of AML-specific signs/ symptoms and impacts based on the literature review. Each patient was given an opportunity to spontaneously report concepts as well as react to the list of pre-identified concepts. Thus, researchers also added any signs/symptoms and impacts spontaneously mentioned by patients that were not already present on the forms. Each sign/symptom and impact was assigned a code. Sub-codes were created to capture whether the sign/symptom or impact was mentioned unaided or aided. Each transcript was analyzed to determine whether the sign/ symptom or impact was mentioned by each respondent based on content analysis of their verbatim responses, and whether this mention was aided or unaided. Disturbance rating codes were created for each sign/symptom and impact and coded for each respondent who mentioned the sign/symptom or impact.

The frequencies and descriptive statistics were evaluated using Microsoft Excel based on the coded responses to determine the frequency of mentions (participant statement), whether the mentions were aided or unaided, and the median disturbance rating of each sign/ symptom or impact. This analysis was conducted and reported separately for both US and Japan interviews.

\section{RESULTS}

\section{Study Participants}

A total of twenty-three US and eighteen Japanese participants were interviewed (Table 2) in the period between January 23 and March 11, 2014. The overall sample consisted of $37 \%$ males and $63 \%$ females; the median age of those interviewed was 48 years. There were fifteen newly-diagnosed high-intensity participants, nine newly-diagnosed low-intensity participants, nine relapse/refractory participants, and eight post-transplant participants.

Saturation was evaluated retrospectively after forty-one participant interviews. We found that none of the most frequently occurring signs/symptoms or impacts were 
Table 2 Baseline characteristics of all participants interviewed

\begin{tabular}{|c|c|c|c|c|c|}
\hline \multirow[t]{2}{*}{ AML patient subgroup } & \multicolumn{2}{|c|}{ Country } & \multirow{2}{*}{$\begin{array}{l}\text { Totals } \\
N=41\end{array}$} & \multicolumn{2}{|c|}{ Demographics } \\
\hline & $\overline{\text { US }}$ & Japan & & Gender & Median age, years \\
\hline \multirow[t]{2}{*}{ Newly-diagnosed, high-intensity } & 10 & 5 & 15 & $33 \% \mathrm{M}$ & 40 \\
\hline & & & & $66 \% \mathrm{~F}$ & \\
\hline \multirow[t]{2}{*}{ Newly-diagnosed, low-intensity } & 4 & 5 & 9 & $56 \% \mathrm{M}$ & 64 \\
\hline & & & & $44 \% \mathrm{~F}$ & \\
\hline \multirow[t]{2}{*}{ Relapse/refractory } & 3 & 6 & 9 & $44 \% \mathrm{M}$ & 48 \\
\hline & & & & $56 \% \mathrm{~F}$ & \\
\hline \multirow[t]{2}{*}{ Transplant } & 6 & 2 & 8 & $13 \% \mathrm{M}$ & 49 \\
\hline & & & & $87 \% \mathrm{~F}$ & \\
\hline \multirow[t]{2}{*}{ Total } & 23 & 18 & 41 & $37 \% \mathrm{M}$ & 48 \\
\hline & & & & $63 \% \mathrm{~F}$ & \\
\hline
\end{tabular}

$A M L$ acute myeloid leukemia, $F$ female, $M$ male

added after the first interview wave (eight interviews) in the US and the first interview wave in Japan (six interviews). Thus, saturation had been achieved after fourteen participant interviews and additional interviews were conducted to ensure adequate sample sizes in each subgroup.

Forty-eight unique signs/symptoms and twenty-four unique impacts of AML and its treatments were mentioned by US participants (Tables $\mathrm{S} 1$ and $\mathrm{S} 2$ in the supplementary material), and thirty-one unique signs/symptoms and twenty-one unique impacts were mentioned by Japanese participants (Tables S3 and S4 in the supplementary material). The most frequently mentioned signs/symptoms and impacts in each participant subgroup were the same as those mentioned in the overall participant sample (Tables S1-S4). By way of example, the most frequently mentioned sign/symptom in the US, fatigue, was reported by 10/10 newly-diagnosed, high-intensity participants, 4/4 newly-diagnosed, low-intensity participants, $3 / 3$ relapse/refractory participants, and $5 / 6$ post-transplant participants.
In Japan, nausea was the most frequently mentioned sign/symptom and was reported by 5/5 newly-diagnosed, high-intensity participants, 4/5 newly-diagnosed, low-intensity participants, $6 / 6$ relapse/refractory participants, and $2 / 2$ post-transplant participants. Given the similarity of signs/symptoms and impacts reported in each of the subgroups, the results presented below will focus on comparisons and contrasts by region.

Here we present the signs/symptoms and impacts reported by at least $30 \%$ of participants in each subgroup and by $50 \%$ of the overall participant population. Signs/symptoms or impacts reported by less than $30 \%$ of any participant subgroup and by less than $50 \%$ of the overall population are included in the tables in the supplementary material.

\section{Signs/Symptoms and Their Disturbance Effect on Participants' Lives}

The most commonly reported sign/symptom in the US sample was fatigue (95.7\%; Table 3). One US patient said "It [fatigue] was horrible. I 
couldn't do my normal, daily activities. I'm a stay-at-home mom. Even making dinner for my family, even as far as going to the bathroom, was difficult. It took a lot out of you to walk from a very short distance." Other signs/symptoms reported by $\geq 50 \%$ of the US sample included: bruising, weakness, fever, anemia, diarrhea, dizziness, infection, nausea, mouth sores, shortness of breath, bleeding, and headache. Fatigue, fever, infection, nausea, and headache were the symptoms most often mentioned spontaneously by at least half of the respondents.

The most commonly reported symptom in the overall Japanese population was nausea (94.4\%; Table 4). A Japanese participant contextualized their nausea as "I got fatigue and nausea as the side effects of anticancer drugs. Especially [caused] by the radiation therapy." The signs/symptoms reported by $\geq 50 \%$ of the sample from Japan included fatigue, headache, shortness of breath, weakness, vomiting, bleeding, cardiovascular problems, dizziness, fever, poor healing, anemia, diarrhea, constipation, infection, and pain. Those concepts spontaneously reported by at least half of the respondents included fatigue, fever, infection, nausea, and headache.

Plots of disturbance ratings against frequency of concepts reported are presented in Figs. 1, 2, 3 and 4. The highest median disturbance ratings (range 0-10) for US participants were for signs/symptoms of fatigue, fever, infection, and mouth sores (scores of 7-8), followed by those for weakness, diarrhea, dizziness, nausea, shortness of breath, bleeding, headache, swollen/bleeding gums, and poor healing (scores of 5-6). A US patient described his fever and stated, "For about 3 days, I was having this fever and heart rates of about 150 beats per minute. And just felt awful, along with the rapid weight gain. The fever wasn't responding to Tylenol, which was the only thing that they could give me because I had leukemia. I ended up having to be packed in ice packs about every 2 hours for about 3 days straight." Another patient commented on his increased risk of infection noting, "I have susceptibility to infection. With the immune suppression medication, I also don't want to be around crowds if I can avoid it because people equal sickness. The treatment, the immune suppression therapy is a big problem."

The highest median disturbance ratings (range 0-10) for Japan participants were sign/ symptom concepts of shortness of breath (score of 9), fatigue, weakness, infection, and pain (scores of 8 for all), headache and cardiovascular problems (scores of 7), and bleeding, dizziness, fever, and anemia (scores of 5-6). A Japanese patient described fatigue stating, "In the office fatigue was like less physical strength. I felt like I have suddenly aged 20 years."

\section{Impacts and Their Disturbance Effect on Participants' Lives}

The most commonly reported impact in the overall US sample was a decreased ability to maintain social/familial roles, with $91.3 \%$ of participants reporting the concept during interviewing (Table 3). A US patient said, "I can't be the mom I used to be to my kids. That's the worst part. I can't run around and play with them anymore. I used to go outside and play catch with them. I used to play soccer with my son. I get tired out just doing anything with them anymore. I can't go make cookies with my daughter anymore because it hurts to stand up for that long. I get tired out. I mean, it's literally affected everything about my life."

The impacts reported by $\geq 50 \%$ of the US sample included anxiety, decreased ability to function, financial difficulties, memory loss, 
Table 3 US symptom and impact concept frequency and disturbance ratings: number of patients who mentioned a concept and disturbance ratings of those concepts

\begin{tabular}{|c|c|c|}
\hline Core concepts ${ }^{a}$ & $\begin{array}{l}\text { Number }(\% \text { of } N) \text { of patients } \\
\text { mentioning concept }(N=23)(\%)\end{array}$ & $\begin{array}{l}\text { Disturbance } \\
\text { rating }\end{array}$ \\
\hline \multicolumn{3}{|l|}{ Symptom concepts } \\
\hline Fatigue & $22(95.7)$ & 7 \\
\hline Bruising & $18(78.3)$ & 2 \\
\hline Weakness & $18(78.3)$ & 6 \\
\hline Fever & $17(73.9)$ & 8 \\
\hline Anemia & $16(69.6)$ & 2 \\
\hline Diarrhea & $16(69.6)$ & 5 \\
\hline Dizziness & $15(65.2)$ & 5 \\
\hline Infection & $15(65.2)$ & 8 \\
\hline Nausea & $15(65.2)$ & 6 \\
\hline Mouth sores & $14(60.9)$ & 8 \\
\hline Shortness of breath & $13(56.5)$ & 5 \\
\hline Bleeding & $12(52.2)$ & 6 \\
\hline Headache & $12(52.2)$ & 6 \\
\hline \multicolumn{3}{|l|}{ Impact concepts } \\
\hline Decreased ability to maintain social/familial roles & $21(91.3)$ & 8 \\
\hline Anxiety & $20(87.0)$ & 7 \\
\hline Decreased ability to function & $20(87.0)$ & 8 \\
\hline Financial difficulties & $17(73.9)$ & 9 \\
\hline Memory loss & $17(73.9)$ & 6 \\
\hline Problems concentrating & $17(73.9)$ & 5 \\
\hline Depression & $16(69.6)$ & 9 \\
\hline Fear & $15(65.2)$ & 8 \\
\hline Weight loss & $15(65.2)$ & 1 \\
\hline Remission uncertainty & $15(65.2)$ & 8 \\
\hline Appetite loss & $14(60.9)$ & 7 \\
\hline
\end{tabular}

${ }^{a}$ Core concepts are defined as those reported by $\geq 50 \%$ of participants

b Ratings based on a $0-10$ scale ( 0 does not disturb, 10 greatly disturbing)

problems concentrating, depression, fear, weight loss, remission uncertainty, and appetite loss. Those concepts spontaneously reported by at least half of the respondents included decreased ability to maintain social/familial roles and decreased ability to function. A US participant described decreased ability to function as, "I couldn't continue the work I used to do. I used to 
Table 4 Japan symptom and impact concept frequency and disturbance ratings: number of patients who mentioned a concept and disturbance ratings of those concepts

\begin{tabular}{|c|c|c|}
\hline Core concepts $^{\mathrm{a}}$ & $\begin{array}{l}\text { Number }(\% \text { of } N) \text { of patients } \\
\text { mentioning concept }(N=18)(\%)\end{array}$ & $\begin{array}{l}\text { Disturbance } \\
\text { rating }\end{array}$ \\
\hline \multicolumn{3}{|l|}{ Symptom concepts } \\
\hline Nausea & $17(94.4)$ & 4 \\
\hline Fatigue & $16(88.9)$ & 8 \\
\hline Headache & $16(88.9)$ & 7 \\
\hline Shortness of breath & $15(83.3)$ & 9 \\
\hline Weakness & $15(83.3)$ & 8 \\
\hline Vomiting & $14(77.8)$ & 4 \\
\hline Bleeding & $13(72.2)$ & 6 \\
\hline Cardiovascular problems & $12(66.7)$ & 7 \\
\hline Dizziness & $12(66.7)$ & 5 \\
\hline Fever & $12(66.7)$ & 5 \\
\hline Poor healing & $12(66.7)$ & 4 \\
\hline Anemia & $11(61.1)$ & 5 \\
\hline Diarrhea & $11(61.1)$ & 3 \\
\hline Constipation & $9(50.0)$ & 2 \\
\hline Infection & $9(50.0)$ & 8 \\
\hline Pain & $9(50.0)$ & 8 \\
\hline \multicolumn{3}{|l|}{ Impact concepts } \\
\hline Anxiety & $17(94.4)$ & 5 \\
\hline Decreased ability to function & $17(94.4)$ & 6 \\
\hline Remission uncertainty & $17(94.4)$ & 10 \\
\hline Financial difficulties & $14(77.8)$ & 5 \\
\hline Appetite loss & $13(72.2)$ & 4 \\
\hline Decreased ability to maintain social/familial role & $13(72.2)$ & 8 \\
\hline Fear & $12(66.7)$ & 5 \\
\hline Problems concentrating & $11(61.1)$ & 5 \\
\hline Risk of other cancer & $10(55.6)$ & 10 \\
\hline Weight loss & $10(55.6)$ & 3 \\
\hline
\end{tabular}

${ }^{a}$ Core concepts are defined as those reported by $\geq 50 \%$ of participants

b Ratings based on a $0-10$ scale ( 0 does not disturb, 10 greatly disturbing) 


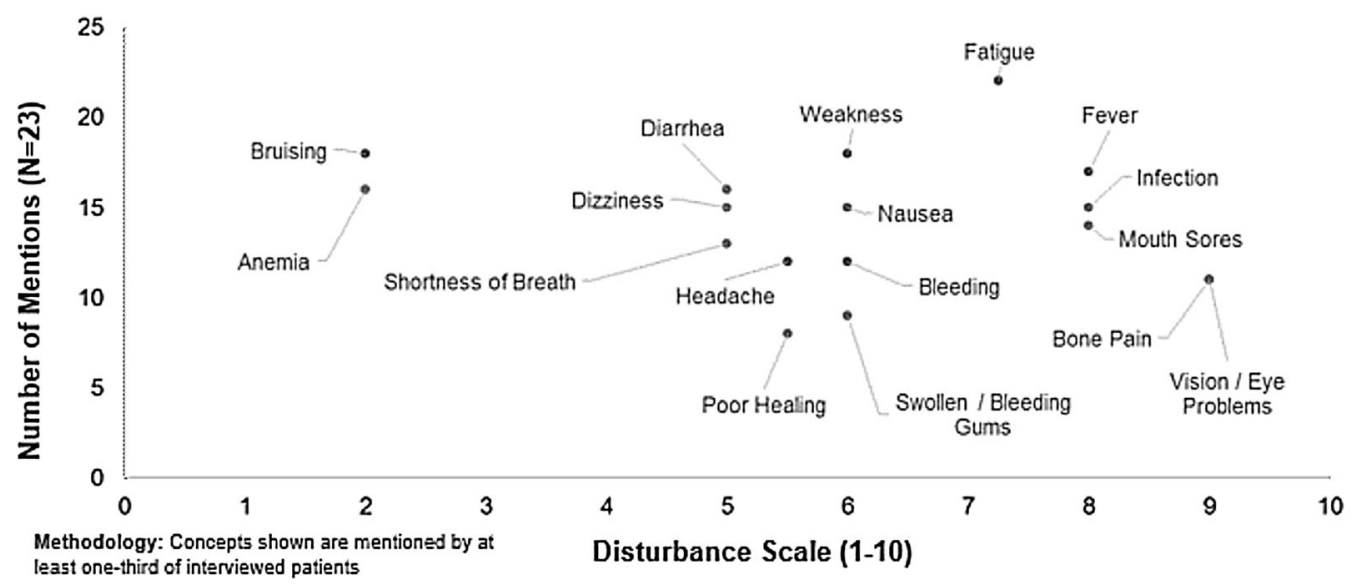

Fig. 1 US acute myeloid leukemia patient symptoms and disturbance ratings from qualitative patient interview data

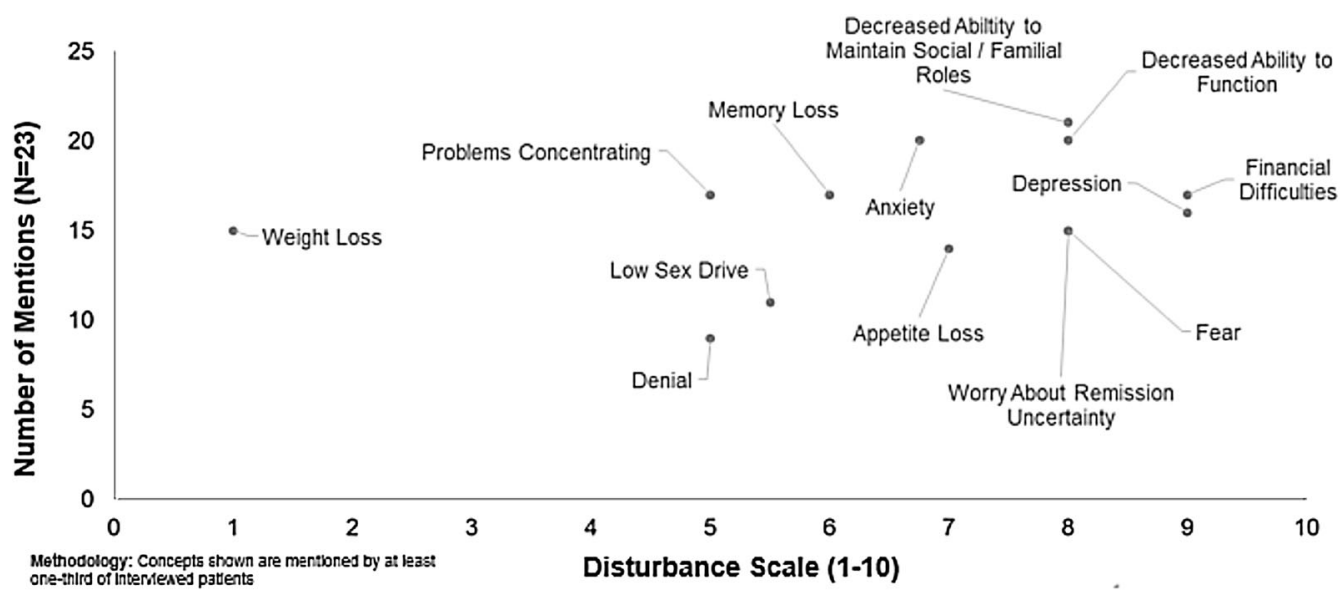

Fig. 2 US acute myeloid leukemia patient impacts and disturbance ratings from qualitative patient interview data

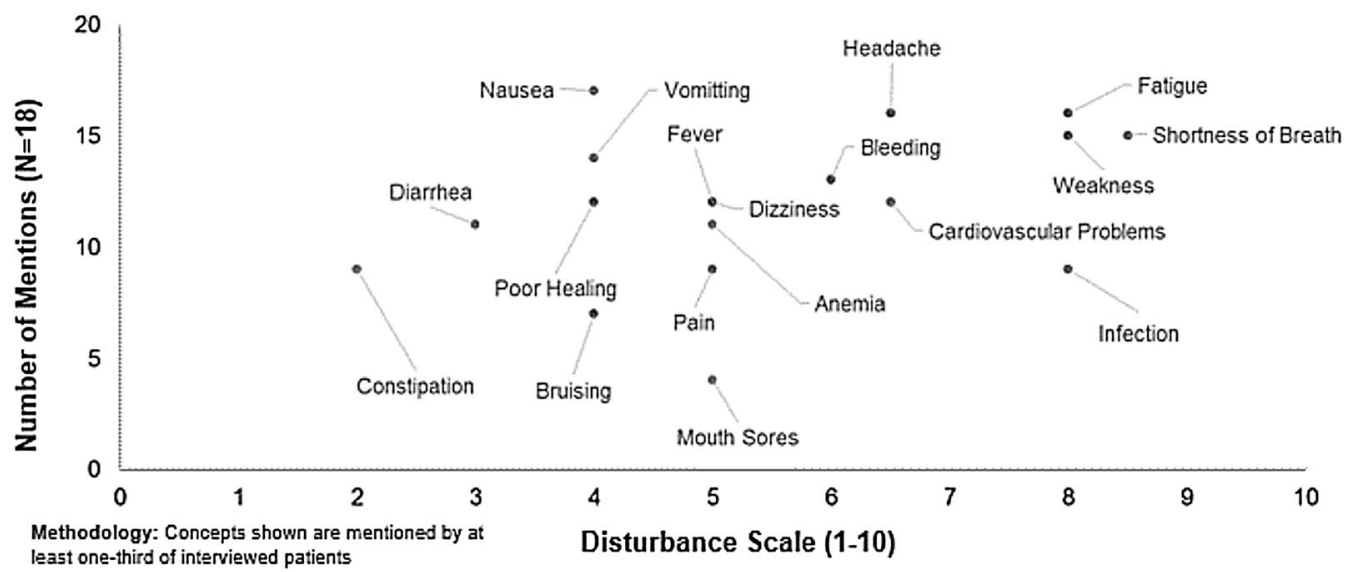

Fig. 3 Japan acute myeloid leukemia patient symptoms and disturbance ratings from qualitative patient interview data 


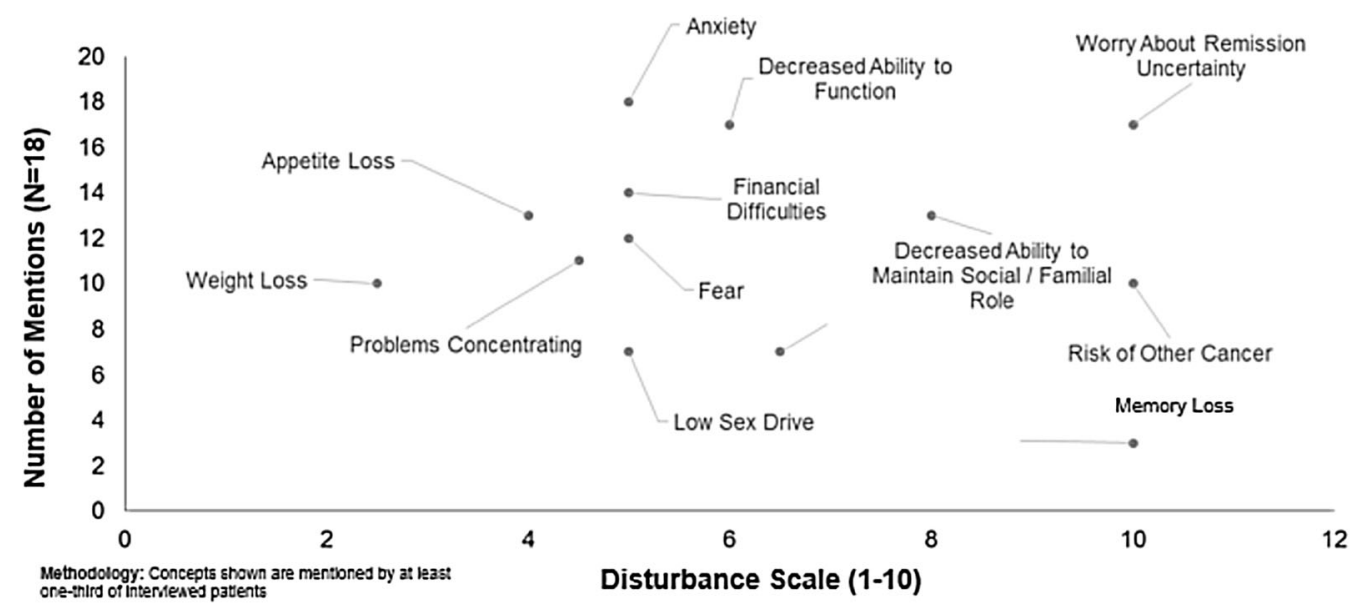

Fig. 4 Japan acute myeloid leukemia patient impacts and disturbance ratings from qualitative patient interview data

be a medical transcriptionist. I couldn't do that anymore because it affected my cognitive abilities and my word recall. I can't do the same work that I did before."

The most commonly reported impact for Japanese participants was anxiety (94.4\%; Table 4). Other impacts reported by $\geq 50 \%$ of the sample from Japan included decreased ability to function, remission uncertainty, financial difficulties, appetite loss, decreased ability to maintain social/familial roles, fear, problems concentrating, risk of other cancer, and weight loss. Decreased ability to function was the only impact spontaneously reported by at least half of the respondents. A Japanese patient described this decreased ability to function as, "I felt I could not work and started to take time off from work. When I got home, I felt like I could not do anything." Anxiety and remission uncertainty were discussed by another Japan participant as, "I felt very anxious [during treatment] because I could not meet my children and four cats... When my doctor started to administer anticancer drugs to me, it eased the symptoms. But I feel I cannot endure the side-effects of anticancer drugs if AML recurs. I am very anxious about it."
The highest median disturbance ratings (range 0-10) for US participants were impact concepts of financial difficulties and depression (scores of 9 for both). A US participant noted that, "It was more just that you're sitting around doing nothing. Couldn't work. Couldn't go anywhere. Not feeling good. I don't think it was bad, but I guess I probably was depressed at times, yeah." These were followed by decreased ability to maintain social/familial roles, decreased ability to function, anxiety, fear, remission uncertainty, and appetite loss (scores of 7-8), problems concentrating and memory loss (scores of 5-6).

The highest median disturbance ratings for Japanese participants (range 0-10) were impact concepts of remission uncertainty and risk of other cancer (scores of 10 for both). These were followed by decreased ability to maintain social/familial role (scores of 7-9), anxiety, decreased ability to function, financial difficulties, fear, and problems concentrating (scores of 5-6). In terms of decreased ability to maintain social/familial role, a Japanese participant said, "She [my wife] may be offended. Our marriage was arranged, so I am afraid that she may start thinking that she made a bad choice." 


\section{DISCUSSION}

This study characterizes patients' experiences of living with AML from their perspective. A key finding is that the most common signs and symptoms were reported consistently across different stages of AML and across two different regions. Patients reported that their most common and disturbing the signs/ symptoms (i.e., fatigue, weakness, and shortness of breath) are related to their most common and disturbing impacts (i.e., anxiety, decreased ability to function, and decreased ability to maintain social/familial roles). For example, patients who are highly disturbed by fatigue and weakness experience a decreased ability to function and maintain their social/familial roles. In addition, US participants seemed to be more comfortable spontaneously discussing their disease, whereas Japan participants needed additional probing to acknowledge some aspects of living with AML. Finally, the signs, symptoms, and impacts reported directly by the patients are similar to those found in the published literature [5-8, 10-13].

Although this study was developed in line with the accepted methodology for this type of research, the sample size was relatively small, resulting in variability in the number of participants per subgroup. Such variability in sample size may explain some of the differences in the relative frequency of concept reporting between the US and Japan participants. We evaluated saturation within country and subgroups and found that it was reached (i.e., no new information was reported after the first wave of interviews). Therefore, in the context of relatively small sampling of subjects, we observed a high degree of consistency in patients' feedback.

In addition, patients from the US and Japan were recruited using different methods. Patients in Japan were clinically confirmed with their diagnosis of AML, whereas patients in the US self-reported their diagnosis. This may result in patients in the US misclassifying their condition. However, we do not believe this occurred as patients were recruited from AML internet sites, were screened appropriately, and confirmed their diagnosis during the interview.

Demographics and clinical characteristics, including comorbidities and details on treatments and concomitant medications, were not collected as part of this study. This may be a limitation to the interpretation of the concepts due to confounding effects-the concepts reported may not necessarily be attributed to the disease, but rather to pre-existing comorbidities or medication side-effects. Although we acknowledge this limitation, we believe this did not significantly impact our findings since major comorbidities such as congestive heart failure, major infections, and hepatic impairment were screened for and there was a consistency in concepts reported among the four different treatment groups.

Finally, most patients could not attribute whether symptoms were related to disease or treatment (e.g., fatigue was a result of AML but was worsened by treatment). While patients were able to describe their symptoms prior to and during treatment, they generally were unable to assess whether changes to the symptoms were a result of the disease worsening or a result of treatment side-effects.

Despite these limitations, the frequency and disturbance of a core set of signs/symptoms (e.g., fatigue, nausea, and shortness of breath) and impacts (e.g., anxiety and remission uncertainty) experienced across the patient sample has implications for both clinical research and healthcare settings. For clinical trial research, our findings suggest that a 
concise tool focusing on this core set of signs/ symptoms and impacts may be appropriate for assessing the most relevant aspects of the patient experience. Further research is necessary to determine whether a single, core tool can be developed that is replicable in broader AML populations across regions other than those assessed in this research study. In the healthcare setting, this research may help providers become more aware of how patients experience the signs/symptoms and impacts of AML. This greater awareness may make providers more likely to ask patients about specific details of their experience and offer interventions to help mitigate the negative impacts of living with AML.

\section{CONCLUSIONS}

These findings are relevant to those living with AML in both the US and Japan. Fatigue, weakness, and shortness of breath emerged as important symptoms, whereas anxiety and decreased ability to function emerged as important impacts. Additionally, these findings are also relevant to those researching AML as they suggest that understanding and measuring the patient experience may be similar regardless of region and patient subgroup, pending additional research in other regions and larger patient subgroups.

\section{ACKNOWLEDGMENTS}

This research was funded by Astellas Pharma Inc., Northbrook, IL, USA. Medical writing and editorial support was provided by Quintiles (New York, NY, USA) and funded by Astellas Pharma Inc. Managerial support was provided by Choice Healthcare Solutions (Chicago, IL, USA) and funded by Astellas Pharma Inc. All named authors meet the International Committee of Medical Journal Editors (ICMJE) criteria for authorship for this manuscript, take responsibility for the integrity of the work as a whole, and have given final approval for the version to be published.

Disclosures. Erin L. Tomaszewski, at the time of the research, was an employee with Quintiles under contract with Astellas Pharma Inc to conduct this research. Catherine E. Fickley is an employee of Quintiles, who conducted the research and prepared the manuscript, under a contract with Astellas Pharma, Inc. LeAnne Maddux is an employee of Quintiles, who conducted the research and prepared the manuscript, under a contract with Astellas Pharma, Inc. Robert Krupnick is an employee of Quintiles, who conducted the research and prepared the manuscript, under a contract with Astellas Pharma, Inc. Erkut Bahceci is an employee of Astellas Pharma Global Development. Jean Paty is an employee of Quintiles, who conducted the research and prepared the manuscript, under a contract with Astellas Pharma, Inc. Floortje van Nooten, at the time of the research, was an employee with Astellas Pharma Europe B.V.

Compliance with Ethics Guidelines. All procedures performed in the studies involving human participants were in accordance with the ethical standards of the institutional and/or national research committee and with the 1964 Helsinki declaration and its later amendments or comparable ethical standards. Informed consent was obtained from all individual participants included in the study.

Open Access. This article is distributed under the terms of the Creative Commons Attribution-NonCommercial 4.0 International 
License (http://creativecommons.org/licenses/ by-nc/4.0/), which permits any noncommercial use, distribution, and reproduction in any medium, provided you give appropriate credit to the original author(s) and the source, provide a link to the Creative Commons license, and indicate if changes were made.

\section{REFERENCES}

1. Acute myeloid leukaemia (AML): Leukaemia Foundation. (n.d.). Retrieved November 17, 2015, from http://www.leukaemia.org.au/blood-cancers/ leukaemias/acute-myeloid-leukaemia-aml. Accessed 17 Nov 2015.

2. UpToDate. (n.d.). Clinical manifestations, pathologic features, and diagnosis of acute myeloid leukemia. Retrieved October 2, 2015, from http://www.uptodate.com/contents/clinicalmanifestations-pathologic-features-and-diagnosis-ofacute-myeloid-leukemia? source=search_result\& search $=$ acute + myeloid + leukemia\&selectedTitle $=2 \%$ 7E150\#H2. Accessed 2 Oct 2015.

3. Wang H-I, Aas E, Howell D, Roman E, Patmore R, Jack A, Smith A. Long-term medical costs and life expectancy of acute myeloid leukemia: a probabilistic decision model. Value Health J Int Soc Pharmacoecon Outcome Res. 2014;17(2):205-14. doi:10.1016/j.jval.2013.12.007.

4. Redaelli A, Botteman MF, Stephens JM, Brandt S, Pashos CL. Economic burden of acute myeloid leukemia: a literature review. Cancer Treat Rev. 2004;30(3):237-47. doi:10.1016/j.ctrv.2003.11.002.

5. Ault PS, Kantarjian HM, Garcia-Manero G, Quintas-Cardama A, Borthakur G, Cortes JE. Symptom burden in acute myeloid leukemia (AML) and myelodysplastic syndrome (MDS). Blood. 2013;122(21):2988. Retrieved from http://www. bloodjournal.org/content/122/21/2988.abstract.

6. Cella D, Jensen SE, Webster K, Hongyan D, Lai J-S, Rosen S, Yount S. Measuring health-related quality of life in leukemia: the Functional Assessment of Cancer Therapy-Leukemia (FACT-Leu) questionnaire. Value Health $\mathrm{J}$ Int Soc Pharmacoecon Outcome Res. 2012;15(8):1051-8. doi:10.1016/j.jval.2012.08.2210.
7. Schumacher A, Kessler T, Büchner T, Wewers D, van de Loo J. Quality of life in adult patients with acute myeloid leukemia receiving intensive and prolonged chemotherapy-a longitudinal study. Leukemia. 1998;12(4):586-92. Retrieved from http://www.ncbi.nlm.nih.gov/pubmed/9557618.

8. UK CR. Acute myeloid leukaemia symptoms. Cancer Research UK. December 3, 2014. Retrieved from http://www.cancerresearchuk.org/about-cancer/type/ aml/about/acute-myeloid-leukaemia-symptoms.

9. Preethi CR. Clinico-hematological study of acutemyeloid leukemias. J Clin Diagn Res: JCDR. 2014;8(4):FC14-7. doi:10.7860/JCDR/2014/7854. 4298 .

10. Hamid G. Acute leukemia clinical presentation. Intech. 2013. Retrieved from http://cdn. intechopen.com/pdfs-wm/44629.pdf. Accessed 24 Aug 2016.

11. Acute myeloid leukemia signs and symptoms, conditions and treatments, UCSF medical center. (n.d.). Retrieved October 16, 2015, from http://www. ucsfhealth.org/conditions/acute_myeloid_leukemia/ signs_and_symptoms.html.

12. Alibhai $\mathrm{SMH}$, Leach $\mathrm{M}$, Kowgier ME, Tomlinson GA, Brandwein JM, Minden MD. Fatigue in older adults with acute myeloid leukemia: predictors and associations with quality of life and functional status. Leukemia. 2007;21(4):845-8. doi:10.1038/sj. leu. 2404576.

13. Oliva EN, Nobile F, Alimena G, Ronco F, Specchia G, Impera S, Latagliata R. Quality of life in elderly patients with acute myeloid leukemia: patients may be more accurate than physicians. Haematologica. 2011;96(5):696-702. doi:10.3324/haematol.2010. 036715.

14. Silveira CC, Gorini MIPC. [Conceptions of the acute myeloid leukemia patient concerning fatigue]. Revista gaúcha de enfermagem/EENFUFRGS. 2009;30(1):40-5. Retrieved from http://www.ncbi. nlm.nih.gov/pubmed/19653554.

15. Food and drug administration. Guidance for industry: patient-reported outcome measures: use in medical product development to support labeling claims. 2009. Retrieved from http://www. fda.gov/downloads/Drugs/Guidances/UCM193282. pdf. Accessed 24 Aug 2015. 